\title{
Direct simulation of weak axisymmetric fountains in a homogeneous fluid
}

\author{
By WENXIAN LIN AND S. W. ARMFIELD \\ Department of Mechanical and Mechatronic Engineering, The University of Sydney, \\ NSW 2006, Australia
}

(Received 5 June 1998 and in revised form 25 August 1999)

The weak axisymmetric fountain that results from the injection of a dense fluid upwards into a large container of homogeneous fluid of lower density is studied numerically. Using a time-accurate finite volume code, the behaviour of fountains with both a uniform and a parabolic profile of the discharge velocity at the source has been investigated. The evolution of the transient fountain flow has been analysed and two distinct stages have been identified. The time series of the passage of the fountain front has been presented and the initial, temporary and final characteristic fountain heights have been determined and scaled with the Froude number at the source. At steady state, the final fountain height and the fountain width are found to be the height and horizontal length scales which provide the full parameterization of the flow in the fountain core. The vertical velocity and temperature on the symmetry axis have been scaled with the height scale and an explicit correlation is also obtained for the former. The radial distributions of both the vertical and horizontal velocities in the zone of self-similarity in the fountain core at steady state have been scaled with the two length scales and empirical correlations have been obtained.

\section{Introduction}

Negatively buoyant jets (often referred to as fountains) are common both in industry and in nature. In any process where a dense fluid is steadily injected vertically upward into a miscible and less dense fluid (or in the reverse case, when lighter fluid is injected vertically downward into a denser ambient fluid) a fountain-type structure forms. When the fountain source is strong, that is when the discharge momentum is relatively large compared to the negative buoyancy, the flow becomes turbulent quite close to the source and is characterized by $F r>1.0$, where $F r$ is the Froude number defined as (Chen \& Rodi 1980; Rodi 1982)

$$
F r=\frac{V_{0}}{\left(R_{0} \sigma_{0}\right)^{1 / 2}},
$$

in which $V_{0}$ is the mean momentum-weighted discharge velocity at the fountain source, defined in $\S 2.1, R_{0}$ is the radius of the source, and $\sigma_{0}$ is the reduced gravity between the fountain and the ambient fluid at the source. For these turbulent fountains, the momentum of the rising denser fluid is reduced gradually by the negative buoyancy until the flow first comes to rest at an initial height above the source. After that, the flow falls back as an annular plunging plume around the upward flow and this downflow continues to mix with the ambient while also interacting turbulently with the upflow which restricts the rise of further fluid and therefore reduces the initial 


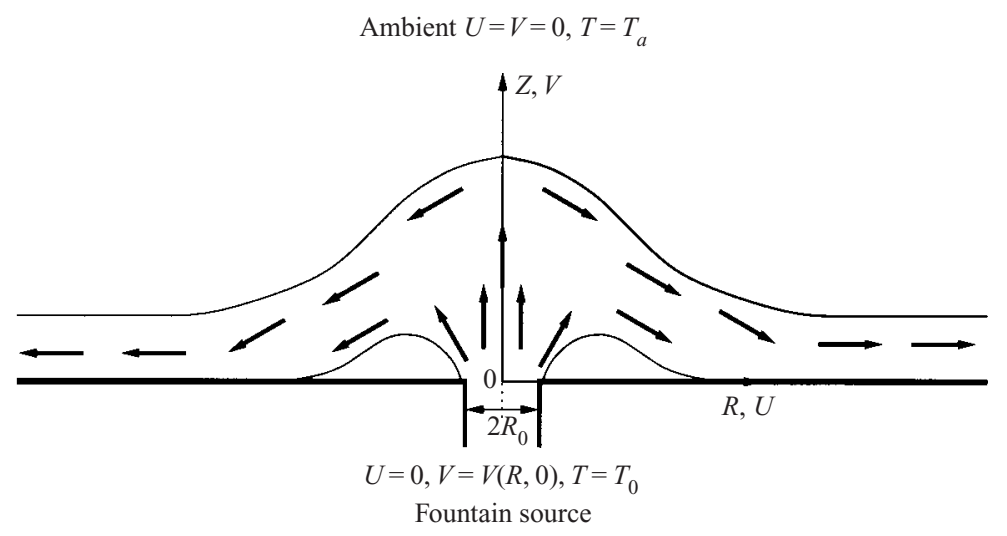

FIGURE 1. A schematic of a weak fountain flow.

fountain height to a final value which is observed to be related to the momentum and buoyancy fluxes at the source (Campbell \& Turner 1989; Baines, Turner \& Campbell 1990; Bloomfield \& Kerr 1998). The schematic drawing in figure 1 by Baines et al. (1990), Baines, Corriveau \& Reedman (1993) is very instructive for understanding this process.

For fountains with a weak source, the discharge momentum of the fountain is equal to or less than the negative buoyancy and the maximum fountain height $Z_{m}$ is of the order of $R_{0}$. The fountain is characterized by a small Froude number $(F r \leqslant 1.0)$. It has no distinguishable upward and downward flows, as shown schematically in figure 1. Instead, the streamlines curve and spread from the source. From dimensional analysis, Baines et al. (1993) argued that in this specific situation,

$$
\frac{Z_{m}}{R_{0}} \sim F r .
$$

No further information is available to determine the exact form of (1.2) and no study has been found that investigated in depth the transient flow patterns of these weak fountains, despite their importance from a fundamental and applied point of view. This motivates us to conduct the current study to investigate numerically the behaviour of weak axisymmetric fountains in a homogeneous fluid.

Weak fountains occur in many environmental and industrial flows. One example is the replenishing of cold salt water at the bottom of solar ponds. Solar ponds are large, shallow bodies of salt water that are arranged so that the temperature gradients are reversed from the normal, i.e. the hottest layers are at the bottom of the ponds (Duffie \& Beckman 1991). This allows their effective use for collection and storage of solar energy. To maximize the efficiency of solar ponds, it is crucial to maintain stable temperature and concentration gradients and to suppress convective flows inside the ponds. Another example is the melting of the roof of a magma chamber, which can lead to weak positively buoyant downward jets as described by Huppert \& Sparks (1988).

There have been numerous studies carried out on the behaviour of turbulent fountains. Although the present study considers weak laminar fountains a brief review of studies of turbulent fountains is given below to provide the theoretical background for the present investigation. Morton $(1959 a, b)$ made the first study of a turbulent fountain in both homogeneous and stratified environments. He analysed the fountain as one case of a forced plume and used the entrainment equations to 
quantify the increasing radius, the decreasing buoyancy and the velocity of dense fluid injected upward into a lighter ambient fluid. Based on the assumption that the flow is controlled by the fluxes of momentum and buoyancy at the source, Turner (1966) demonstrated that dimensional consistency requires that

$$
Z_{m}=C M_{0}^{3 / 4} F_{0}^{-1 / 2},
$$

where $C$ is a constant and $M_{0}$ and $F_{0}$ are the momentum and buoyancy fluxes at the source defined as

$$
M_{0}=\pi R_{0}^{2} V_{0}^{2}
$$

and

$$
F_{0}=\pi \sigma_{0} R_{0}^{2} V_{0}
$$

for a uniform profile. From (1.3), Baines et al. (1990) obtained the following relation:

$$
\frac{Z_{m}}{R_{0} F r}=C .
$$

Turner (1966) found that $C=2.46$ for the final fountain height from a laboratory experiment in which the penetration distance of a salt-water jet injected into fresh water had been measured for $2<F r<30$. The theoretical analysis carried out by Morton (1959a), which included some experimentally determined values, gave $C=2.06$ for the initial fountain height while no result was given for the final fountain height. Therefore, both the theoretical and experimental results show that $Z_{m}$ is considerably greater than $R_{0}$ for a turbulent fountain. Abraham (1967) argued that the assumption made by Morton (1959a), that the vertical flux of a tracer being contained in the jet is constant from the source to $Z_{m}$, is not realistic, and suggested that near $Z_{m}$ the vertical flux of jet fluid and the vertical flux of a tracer carried by the jet decrease with height. Abraham (1967) then proposed an analytical solution which takes this into consideration and obtained $C=2.74$ for the final fountain height which is $111 \%$ of the value obtained experimentally by Turner (1966) for the final fountain height, as compared to $84 \%$ obtained by Morton $(1959 a, b)$ for the initial height.

Seban, Behnia \& Abreau (1978) studied a heated turbulent air jet discharged downward and found that centreline temperatures and $Z_{m}$ may be predicted adequately by constant property theories for the downward flow alone and obtained $C=2.35$ for the final fountain height for $\mathrm{Fr}>30$. This same result was also obtained by Mizushina et al. (1982) who investigated experimentally the behaviour of a vertical turbulent fountain in a uniform fluid and showed that the width of the upflow depends linearly on the distance from the source, and that the time-averaged velocities and temperatures as well as the intensities of their fluctuations at the jet axis can be well correlated with the same scaling law as that used for the buoyant jet. However, they found that the radial distributions show no similarity profiles for these quantities. Detailed information about the earlier theoretical and experimental studies on the behaviour of turbulent fountains can be found in the reviews on turbulent jets and plumes by Turner (1973, 1986), Fischer et al. (1979), Chen \& Rodi (1980), Rodi (1982), and List (1982).

Recently, Campbell \& Turner (1989) and Baines et al. (1990) made further investigations into turbulent fountains. Baines et al. (1990) combined the experimental results of Turner (1966) and Seban et al. (1978) with theirs and found that $C$ is still 2.46 for the final fountain height. An experimental and numerical investigation of the density distribution produced in a container by a turbulent fountain was conducted 
(a)

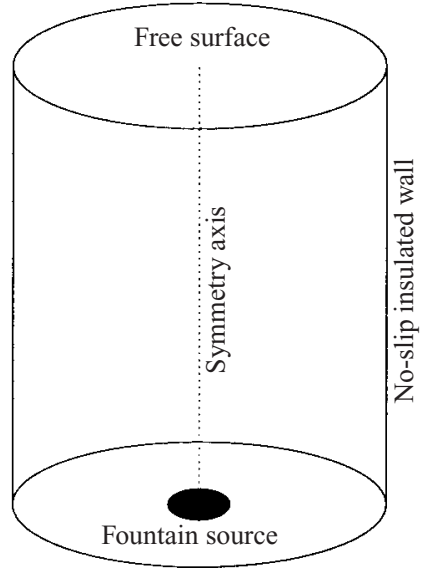

(b)

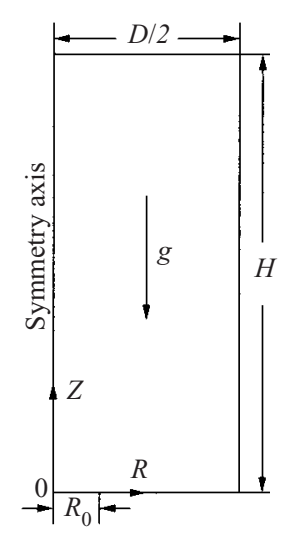

FIGURE 2. (a) Sketch of the system and (b) the computational domain.

by Baines et al. (1993) to evaluate the effect of the forced vertical motion of the environment. Bloomfield \& Kerr (1998) studied both axisymmetric and line turbulent fountains in a stratified fluid using a combination of dimensional arguments and laboratory experiments and found that after the fountain reaches the initial height at which the flow first comes to rest the subsequent downflow may either spread along the base of the tank or intrude at an intermediate height in the environment, depending on the strength of the stratification and the fluxes of momentum and buoyancy at the source.

In this study, we investigate numerically the behaviour of weak axisymmetric fountains issuing vertically into a quiescent homogeneous ambient fluid. In $\S 2$, we outline the numerical method in which a time-accurate finite volume code is used. In $\S 3$, numerical results for $F r=1.0$ are presented to provide qualitative observations on the evolution of the transient fountain flow. Quantitative observations are then presented to show the time series of the passage of the fountain front, to determine the characteristic length scales, that is the fountain height and fountain width which describe the fountain flow at steady state, and to provide a critical value of $F r$ separating weak and strong fountains. In $\S 4$, the velocity and temperature distributions inside the fountain core at steady state are analysed and scaled using the two length scales. Finally, the main conclusions are summarized in $\S 5$.

\section{Numerical method}

\subsection{Governing equations}

Under consideration is a vertical circular container of height $H$ and diameter $D$ containing a Newtonian fluid initially at rest and at temperature $T_{a}$ (figure 2); the sidewall is non-slip and insulated and the top is open. In the bottom centre, an orifice with radius $R_{0}$ is used as the fountain source. The remaining bottom region is a rigid non-slip insulated boundary. At time $t=0$, a stream of fluid at $T=T_{0}<T_{a}$ is injected impulsively into the container from the source to initiate the fountain and this discharge is maintained thereafter. Due to the symmetry of the system geometry and the boundary and initial conditions, the fountain flow in the container is axisymmetric, which ensures that only the computational domain sketched in figure $2(b)$ is needed. 
The weak fountain discharge enables us to assume a laminar flow. At the fountain source, both parabolic and uniform velocity profiles have been used. The parabolic profile is defined as

$$
V(R, 0)=V_{m}\left[1-\left(\frac{R}{R_{0}}\right)^{2}\right] \quad \text { for } 0 \leqslant R \leqslant R_{0},
$$

where $V_{m}$ is the maximum vertical velocity at the source. The uniform velocity profile is $V(R, 0)=V_{0}$ for $0 \leqslant R \leqslant R_{0}$. The uniform and parabolic profiles are considered comparable when they have the same momentum flux, which requires that $V_{m}=\sqrt{3} V_{0}$. For the parabolic profile the mean momentum-weighted discharge velocity is defined to be $V_{0}=V_{m} / \sqrt{3}$, and therefore the uniform and parabolic fountains will have the same Froude number when their momentum fluxes are equal.

The flow is described by the Navier-Stokes equations and the temperature equation, with the Boussinesq assumption allowing their incompressible forms to be used. The equations are written in conservative, non-dimensional form in cylindrical coordinates as follows:

$$
\begin{gathered}
\frac{1}{r} \frac{\partial}{\partial r}(r u)+\frac{\partial v}{\partial z}=0, \\
\frac{\partial u}{\partial \tau}+\frac{1}{r} \frac{\partial}{\partial r}(r u u)+\frac{\partial}{\partial z}(v u)=-\frac{\partial p}{\partial r}+\frac{1}{R e}\left\{\frac{\partial}{\partial r}\left[\frac{1}{r} \frac{\partial}{\partial r}(r u)\right]+\frac{\partial^{2} u}{\partial z^{2}}\right\}, \\
\frac{\partial v}{\partial \tau}+\frac{1}{r} \frac{\partial}{\partial r}(r u v)+\frac{\partial}{\partial z}(v v)=-\frac{\partial p}{\partial z}+\frac{1}{R e}\left[\frac{1}{r} \frac{\partial}{\partial r}\left(r \frac{\partial v}{\partial r}\right)+\frac{\partial^{2} v}{\partial z^{2}}\right]+\frac{1}{F r^{2}} \theta, \\
\frac{\partial \theta}{\partial \tau}+\frac{1}{r} \frac{\partial}{\partial r}(r u \theta)+\frac{\partial}{\partial z}(v \theta)=\frac{1}{\operatorname{RePr}}\left[\frac{1}{r} \frac{\partial}{\partial r}\left(r \frac{\partial \theta}{\partial r}\right)+\frac{\partial^{2} \theta}{\partial z^{2}}\right] .
\end{gathered}
$$

In these equations $r$ and $z$ are the dimensionless horizontal and vertical distances from the left bottom corner of the domain, $u$ and $v$ the dimensionless $r$-velocity and $z$-velocity components, $p$ the dimensionless pressure, $\tau$ the dimensionless time, $\theta$ the dimensionless temperature, $R e$ and $P r$ the Reynolds number and the Prandtl number.

$R e$ and $P r$ are defined respectively as follows:

$$
R e=\frac{V_{0} R_{0}}{v},
$$

and

$$
\operatorname{Pr}=\frac{v}{\kappa},
$$

where $\kappa$ and $v$ are the conductivity and kinematic viscosity of the fluid respectively.

Non-dimensional quantities are obtained as follows:

$$
\left.\begin{array}{l}
r=\frac{R}{R_{0}}, \quad z=\frac{Z}{R_{0}}, \quad u=\frac{U}{V_{0}}, \quad v=\frac{V}{V_{0}}, \\
\tau=\frac{t}{\left(R_{0} / V_{0}\right)}, \quad p=\frac{P}{\rho V_{0}^{2}}, \quad \theta=\frac{T-T_{a}}{T_{0}-T_{a}},
\end{array}\right\}
$$

where $R$ and $Z$ are the dimensional horizontal and vertical distances from the left bottom corner of the domain, $U$ and $V$ the dimensional $R$-velocity and $Z$-velocity components, $P$ the dimensional pressure, $t$ the dimensional time, $T$ the dimensional temperature, and $\rho$ the fluid density. 
The appropriate initial and boundary conditions are

$$
u=v=0, \quad \theta=0 \quad \text { at all } r, z \text { and } \tau<0 ;
$$

and

$$
\begin{aligned}
& u=0, \frac{\partial v}{\partial r}=0, \frac{\partial \theta}{\partial r}=0 \quad \text { on } r=0,0 \leqslant z \leqslant \frac{H}{R_{0}} ; \\
& u=v=0, \frac{\partial \theta}{\partial r}=0 \quad \text { on } r=\frac{D}{2 R_{0}}, 0 \leqslant z \leqslant \frac{H}{R_{0}} ; \\
& u=0, v=v(r, 0), \theta=-1 \quad \text { on } 0 \leqslant r \leqslant 1, z=0 \text {; } \\
& u=v=0, \frac{\partial \theta}{\partial z}=0 \quad \text { on } 1<r \leqslant \frac{D}{2 R_{0}}, \quad z=0 ; \\
& \frac{\partial u}{\partial z}=\frac{\partial v}{\partial z}=\frac{\partial \theta}{\partial z}=0 \quad \text { on } 0 \leqslant r \leqslant \frac{D}{2 R_{0}}, \quad z=\frac{H}{R_{0}}, \tau \geqslant 0 .
\end{aligned}
$$

In this study, $H$ has been chosen to be large enough with respect to $R_{0}$ so that the upper $\left(z=H / R_{0}\right)$ boundary has little influence on the fountain flow and that the assumption of parallel flow conditions at the upper boundary in (2.10) is appropriate.

\subsection{Discretization}

Because of the large variations in length scales it is necessary to use a mesh that concentrates points in the fountain region and in the boundary layers and is relatively coarse in other regions. To construct this, a uniform, fine mesh is employed in the fountain region in the $r$-direction and a stretched mesh is distributed in the remaining region both in the $r$ - and $z$-direction. In this study, $H / R_{0}=D /\left(2 R_{0}\right)=10$ and $\Delta r_{w}=\Delta z_{w}=1.25 \times 10^{-2}$ are used, where $\Delta r_{w}$ and $\Delta z_{w}$, non-dimensionalized by $R_{0}$, are the first grid sizes nearest the symmetry axis in the $r$-direction and nearest the bottom floor in the $z$-direction respectively, which results in 80 uniform grid points in the fountain region in the $r$-direction. In the non-fountain region in the $r$-direction, the grid points are distributed symmetrically with respect to $0.5\left(D /\left(2 R_{0}\right)-1\right)$. In the $z$-direction, the grid points are distributed symmetrically with respect to $0.5 H / R_{0}$. The grid point just beyond the fountain region or nearest the wall or floor is located at a half of $\Delta r_{w}$ or $\Delta z_{w}$ away from the boundary. Subsequently, the mesh expands at a rate of $7.6 \%$ up to $r=0.1\left(D /\left(2 R_{0}\right)-1\right)$ or $z=0.1 H / R_{0}$. Beyond $r=0.1\left(D /\left(2 R_{0}\right)-1\right)$ or $z=0.1 H / R_{0}$, the mesh size expansion rate decreases at a rate of $10 \%$ until it reaches zero, resulting in a constant coarse mesh in the interior of the non-fountain region. This mesh construction generates $187 \times 115$ volumes in the computational domain shown in figure $2(b)$.

The equations are discretized on a non-staggered mesh in which all variables are stored at the same grid locations. The method of obtaining the pressure and satisfying continuity is similar to the SIMPLE schemes used with the conventional staggered mesh (Patankar 1980). To enable this approach to be used with a non-staggered mesh, regularizing terms are included in the Poisson equation for the pressure. Without the inclusion of these regularizing terms the scheme would lead to an odd-even splitting of the pressure, with a resulting degradation and ultimate collapse of the solution. The regularizing terms have the effect of ensuring the discrete scheme is strongly elliptic, while without them the scheme is non-elliptic. Comparisons between staggered and non-staggered solutions indicate both schemes have an equivalent accuracy (Armfield 1991). The advantage of using the non-staggered scheme is that all variables have 
the same discrete operators. With a staggered non-uniform mesh a different operator must be used for each of the velocities and the scalar variables. Hence the use of the non-staggered approach leads to a considerable saving in programming and computer time.

Finite volumes are used to convert differential terms in the governing equations to differences as follows. All second derivatives and linear derivatives are approximated by second-order central differences. The convective terms are approximated by a QUICK scheme (Leonard 1979). The discretization produces the usual fringed block tridiagonal matrix operator, one for each of the momentum, temperature and pressure equations. These are solved using an alternating-direction-implicit iterative method. The scheme is described in detail by Armfield $(1991,1994)$ and has been previously used for the simulation of buoyancy-dominated and stratified flows (Patterson \& Armfield 1990; Armfield \& Patterson 1992; Armfield \& Debler 1993; Armfield 1994).

\subsection{Time integration}

A second-order Crank-Nicholson predictor-corrector scheme is used for the time integration of the transport equations in the following way. First, all variables are known at time $\tau=n \Delta \tau$, where $\Delta \tau$ is the time step non-dimensionalized by $R_{0} / V_{0}$. Secondly, the temperature equation (2.5) is inverted to obtain an initial approximation to $\theta^{n+1}$, and using this value the two momentum equations (2.3) and (2.4) are inverted, using an estimated pressure field, to obtain a first approximation to $u^{n+1}$ and $v^{n+1}$. A pressure correction equation derived from equation (2.2) is then solved to enforce continuity. Finally new estimates of $u^{n+1}$ and $v^{n+1}$ are obtained. This procedure is repeated until a preset divergence convergence criterion of $1 \times 10^{-4}$ is met.

\subsection{Grid independence}

To test the grid independence of the scheme the solutions have been obtained on the basic mesh of $187 \times 115$ defined above and on two additional fine meshes, with the following parameters. In the first fine mesh, $\Delta r_{w}=\Delta z_{w}=6.25 \times 10^{-3}$ but the grid expansion factor and the time step are not changed, which gives a mesh of $248 \times 155$. In the second fine mesh, $\Delta r_{w}=6.25 \times 10^{-3}, \Delta z_{w}=3.125 \times 10^{-3}$ and $\Delta \tau=0.5 \times 10^{-4}$ which is half of that used in the basic mesh of $187 \times 115$ but the same grid expansion factor is still used. This gives a mesh of $248 \times 178$. Figure 3 shows the numerically simulated temperature and velocity profiles for the three mesh sizes. It is clear from this figure that the variation between the three representations is very small, indicating that the basic mesh of $187 \times 115$ is free of grid- and time-step-dependent errors.

$\operatorname{Pr}=7, \operatorname{Re}=200, \Delta \tau=1 \times 10^{-4}, H=10 R_{0}$ and $D=20 R_{0}$ are used in all the numerical simulations in this study.

\section{Evolution of transient fountain flow}

\subsection{Qualitative observations}

In figure 4 , a visualization of a typical time evolution of the numerically simulated transient temperature contours is presented for $F r=1.0$ with the uniform profile, providing an overview of the evolution of the weak fountain flow. After the discharge is initiated, it is observed that the momentum of the rising fluid is gradually reduced by the negative buoyancy force until the front of the flow first comes to rest at an initial characteristic fountain height, $z_{m i}$ which is non-dimensionalized by $R_{0}$, approximately at $\tau=3.8$. At the same time, the rising flow begins to spread outwards 

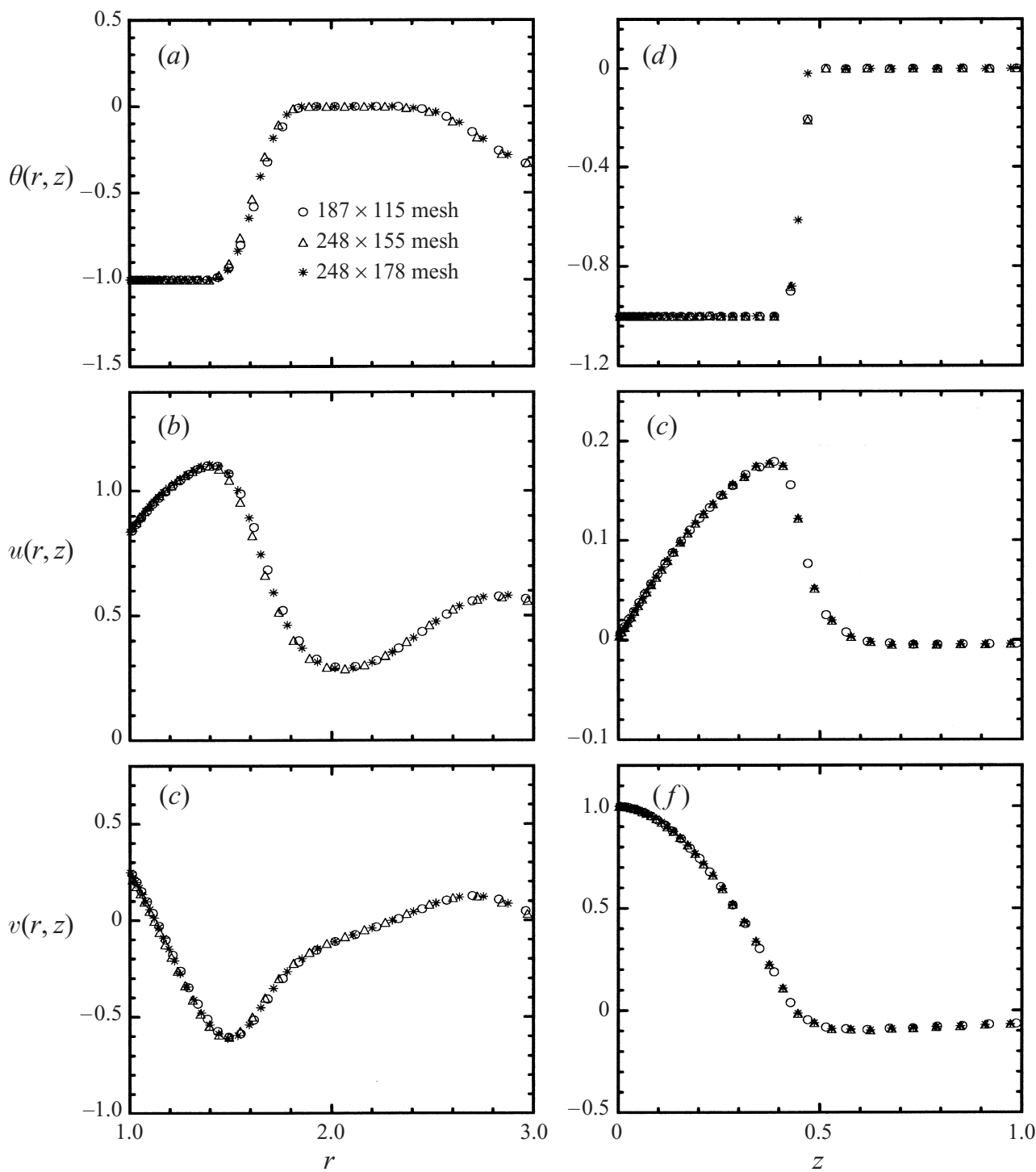

FIGURE 3. Comparison of the numerical results with the $187 \times 115$ basic mesh, $248 \times 155$ and $248 \times 178$ finer meshes: $(a)$ temperature, $(b)$ horizontal velocity and $(c)$ vertical velocity profiles at $z=0.2 ;(d)$ temperature, $(e)$ horizontal velocity and $(f)$ vertical velocity profiles at $r=0.2$ when $\tau=1.0$, respectively.

immediately after discharge due to its reduced velocity and interaction with the ambient fluid. The downflow which is formed after the fountain front reaches $z_{m i}$ continues to interact with the environment while also interacting with the upflow. This interaction restricts the rise of further fluid and therefore reduces $z_{m i}$ to a smaller value, $z_{m t}$, which we refer to as the temporary characteristic fountain height. As the downflow fluid always remains denser than the ambient fluid, it must spread along the bottom floor of the container, resulting in the formation of an eddy in the region bounded by the upflow, downflow and the floor and an intrusion moving outwards along the floor. Initially this eddy contains ambient fluid which is gradually 


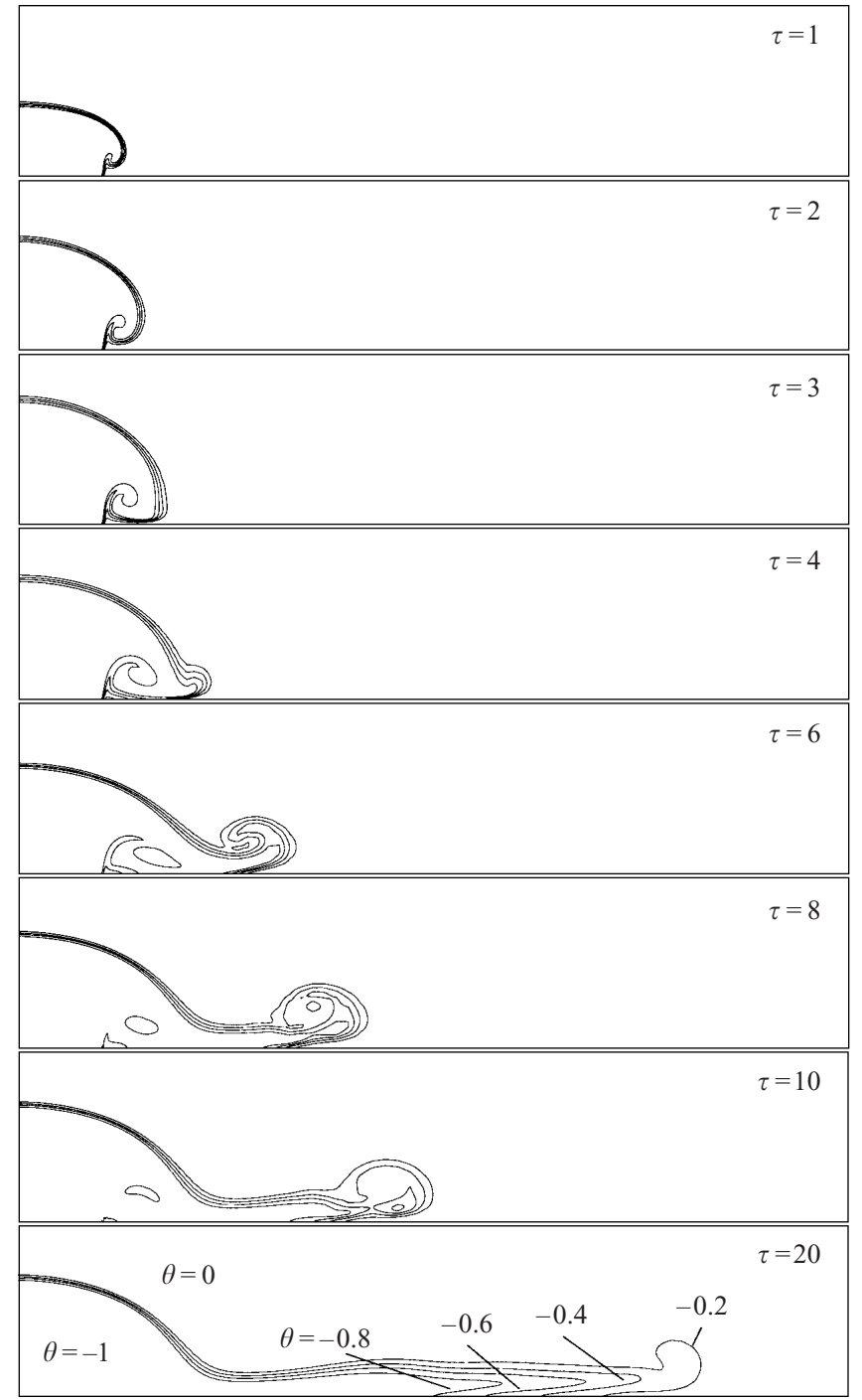

FIGURE 4. A typical time evolution of the transient temperature contours for $F r=1.0$ with the uniform profile.

dissipated as the flow develops. The fountain front stays at $z_{m t}$ for a short time before it rises again. The second rise brings the fountain front to a height $z_{m f}$, which is less than $z_{m i}$, at $\tau=8.8$ and it stays at this height thereafter. After the fountain front reaches $z_{m f}$ the upflow and downflow are steady and the only unsteady flow is the spreading of the intrusion along the bottom floor of the container. Therefore, $z_{m f}$ is the final characteristic fountain height. This flow evolution is similar to that observed for turbulent fountains (Turner 1966; Campbell \& Turner 1989; Baines et al. 1990; and Bloomfield \& Kerr 1998). Qualitatively similar flow patterns are also observed in the weak fountains when the discharge velocity at the source has the parabolic profile represented by (2.1), although there are quantitative differences for the two profiles. 

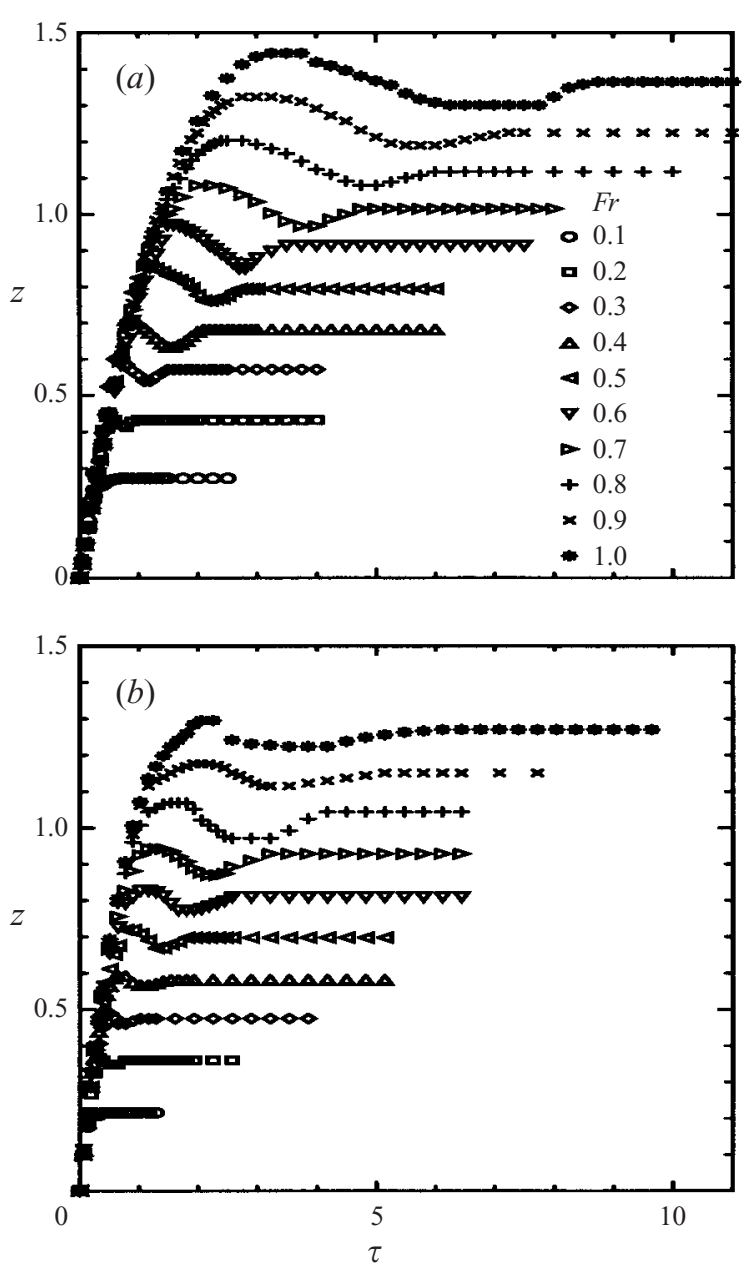

FiguRE 5. Passage of the fountain front for a series of $F r$ with $(a)$ the uniform profile and $(b)$ the parabolic profile.

\subsection{Quantitative observations}

\subsubsection{Time series of the passage of the fountain front}

In figure 5 , the time series of the passage of the fountain front are presented for a series of $F r$ in the range $0.1 \leqslant F r \leqslant 1.0$, where the fountain front is determined as the lowest $z$-location at which the temperature is that of the ambient fluid. The qualitative observations from figure 4 are confirmed by the results shown in this figure.

The dimensional times for the fountain front to reach the initial, temporary and final heights are denoted subsequently as $t_{m i}, t_{m t}$ and $t_{m f}$ while their corresponding dimensionless times are denoted as $\tau_{m i}, \tau_{m t}$ and $\tau_{m f}$ respectively.

\subsubsection{Characteristic fountain heights}

As argued by Baines et al. (1993), dimensional analysis requires that equation (1.2) is valid for weak fountains. To provide detailed information and determine an explicit form of (1.2) for weak fountains, we present in figure 6 the numerically simulated initial, temporary and final heights plotted against $F r$ in the range $0.1 \leqslant F r \leqslant 1.0$ for 

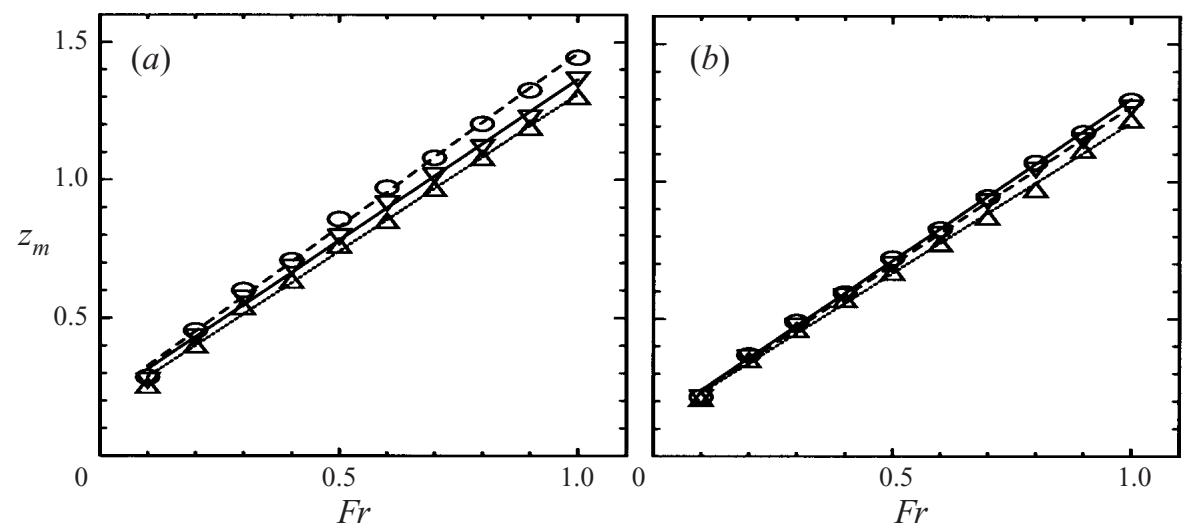

FIGURE 6. Initial, temporary and final heights plotted against $F r$ with $(a)$ the uniform profile and $(b)$ the parabolic profile: $\bigcirc, \triangle, \nabla$, numerically simulated initial, temporary and final heights; $\cdots \cdots,---$, linear-fit initial, temporary and final heights respectively.

\begin{tabular}{|c|c|c|c|c|c|c|}
\hline \multirow[b]{2}{*}{$z_{m}$} & \multicolumn{3}{|c|}{ Uniform } & \multicolumn{3}{|c|}{ Parabolic } \\
\hline & $a_{0}$ & $a_{1}$ & $R C$ & $a_{0}$ & $a_{1}$ & $R C$ \\
\hline$z_{m i}$ & $0.201 \pm 0.014$ & $1.258 \pm 0.023$ & 0.9987 & $0.122 \pm 0.008$ & $1.178 \pm 0.013$ & 0.9995 \\
\hline$z_{m t}$ & $0.173 \pm 0.011$ & $1.137 \pm 0.018$ & 0.9989 & $0.119 \pm 0.011$ & $1.096 \pm 0.017$ & 0.9991 \\
\hline$z_{m f}$ & $0.198 \pm 0.015$ & $1.165 \pm 0.024$ & 0.9983 & $0.119 \pm 0.005$ & $1.155 \pm 0.011$ & 0.9997 \\
\hline
\end{tabular}

both the uniform and parabolic profiles. A linear fit of the form

$$
z_{m}=a_{0}+a_{1} F r
$$

has been obtained for each set of data, where $z_{m}$ represents $z_{m i}, z_{m t}$ and $z_{m f}$ respectively and the values of the constants $a_{0}$ and $a_{1}$, obtained by regression from the numerical results, are listed in table 1 . In the table, $R C$ represents the regression coefficient of the correlation.

As can be seen in figure 6 the linear fit provides a good representation of the data for $0.2 \leqslant F r \leqslant 1.0$ although some departure is observed at $F r=0.1$. As $F r$ goes to zero the linear fit does not go to zero, which is clearly not physical.

When $\mathrm{Fr}>1.0$, numerical results show that the characteristic fountain height increases rapidly, not in accordance with (3.1), as shown in figure 7. In this case, the effect of the fountain discharge momentum at the source dominates the buoyancy and the entrainment between the fountain and the surrounding ambient fluid becomes important. Fountains with $\mathrm{Fr}>1$ are regarded as strong fountains and have different scaling relations and are beyond the scope of this study.

\subsubsection{Time scales for the fountain front}

The fountain flow is governed by the momentum flux $M_{0}$ and the buoyancy flux $F_{0}$ at the source. Therefore, $t_{m}$ can be scaled in terms of combinations of these two fluxes, that is

$$
t_{m}=C M_{0}^{m} F_{0}^{n}
$$

where $C, m$ and $n$ are constants and $t_{m}$ denotes $t_{m i}, t_{m t}$ and $t_{m f}$ respectively. 


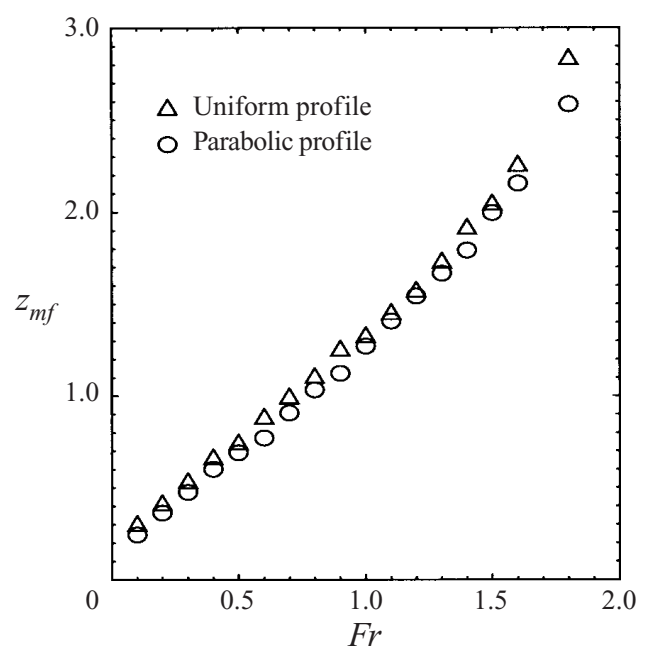

FIGURE 7. $z_{m f}$ plotted against $F r$ for $0.1 \leqslant F r<2.0$.
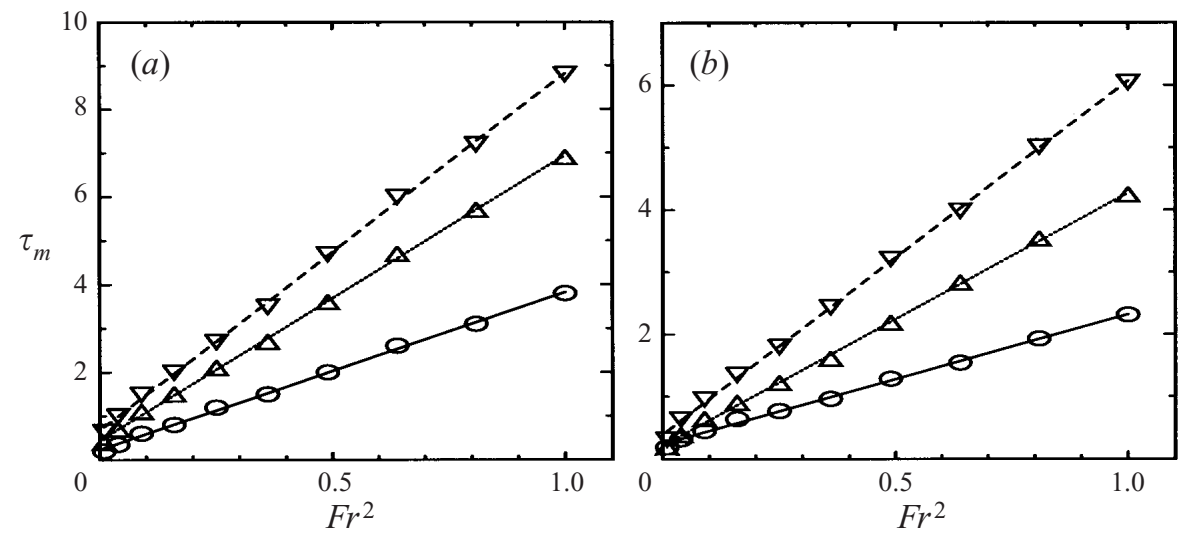

FIGURE 8. Times for the fountain front to reach the initial, temporary and final heights plotted against $F r^{2}$ with $(a)$ the uniform profile and $(b)$ the parabolic profile: $\bigcirc, \triangle, \nabla$, numerically simulated times to reach the initial, temporary and final heights; __ , $\cdots \cdots,----$, linear-fit times to reach the initial, temporary and final heights respectively.

Inserting (1.4) and (1.5) into (3.2) gives

$$
t_{m} \sim \frac{M_{0}}{F_{0}} \sim \frac{R_{0}}{V_{0}} F^{2} .
$$

Hence, $\tau_{m}$ is expected to scale in terms of $F r$ as

$$
\tau_{m}=\frac{t_{m}}{\left(R_{0} / V_{0}\right)} \sim F r^{2} .
$$

Figure 8 shows the numerically simulated $\tau_{m}$ plotted against $F r^{2}$ for $0.1 \leqslant F r \leqslant 1.0$. It is clearly seen that the numerical results confirm the scaling, which is well predicted by the following explicit form:

$$
\tau_{m}=a_{0}+a_{1} F r^{2}
$$




\begin{tabular}{|c|c|c|c|c|c|c|}
\hline \multirow[b]{2}{*}{$\tau_{m}$} & \multicolumn{3}{|c|}{ Uniform } & \multicolumn{3}{|c|}{ Parabolic } \\
\hline & $a_{0}$ & $a_{1}$ & $R C$ & $a_{0}$ & $a_{1}$ & $R C$ \\
\hline$\tau_{m i}$ & $0.232 \pm 0.025$ & $3.592 \pm 0.049$ & 0.9993 & $0.242 \pm 0.019$ & $2.077 \pm 0.039$ & 0.9986 \\
\hline$\tau_{m t}$ & $0.435 \pm 0.033$ & $6.506 \pm 0.066$ & 0.9996 & $0.316 \pm 0.022$ & $4.055 \pm 0.043$ & 0.9995 \\
\hline$\tau_{m f}$ & $0.669 \pm 0.039$ & $8.146 \pm 0.078$ & 0.9996 & $0.392 \pm 0.028$ & $5.678 \pm 0.058$ & 0.9995 \\
\hline
\end{tabular}
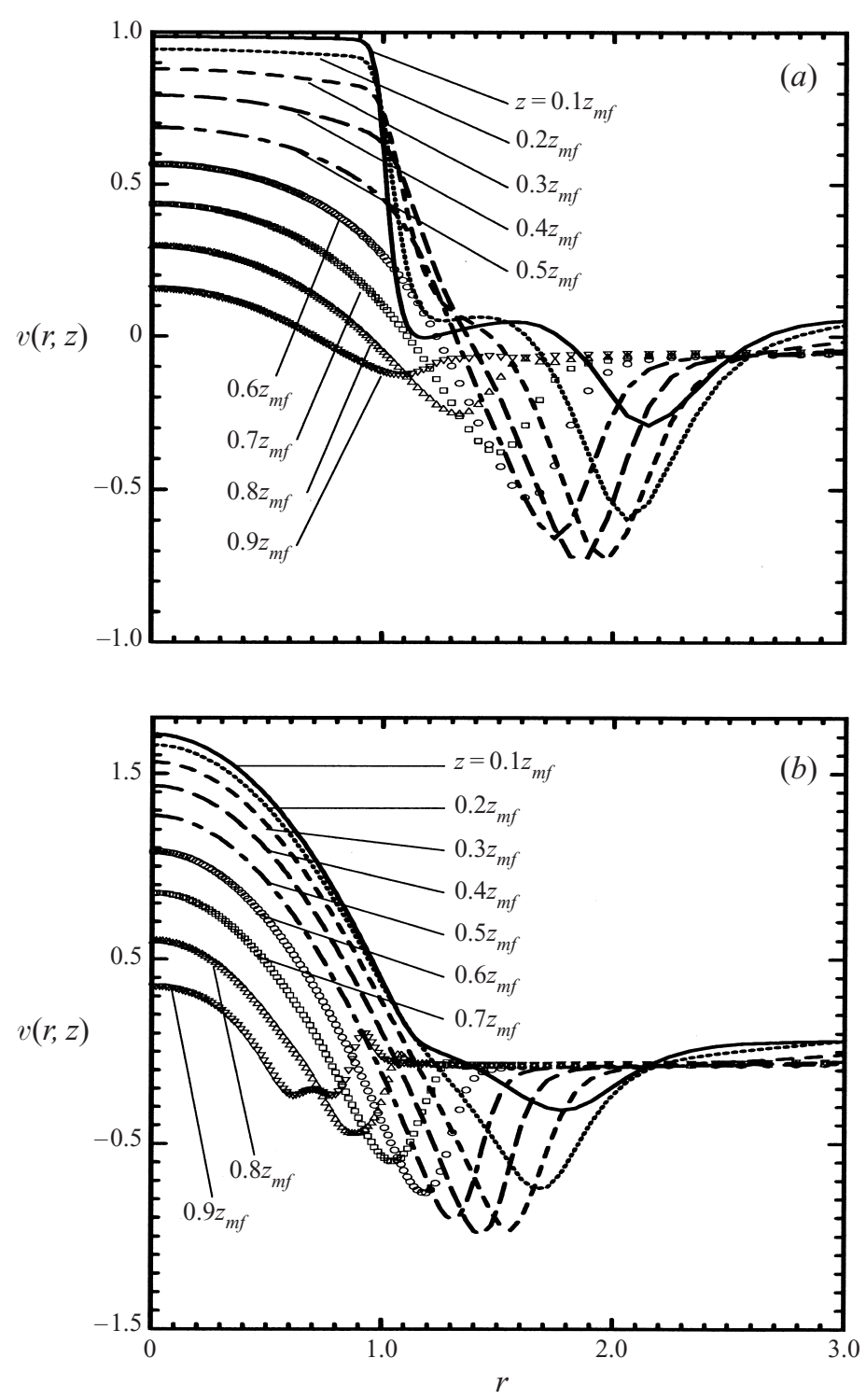

FIGURE 9. Radial distribution of $v(r, z)$ at different heights for $(a)$ the uniform profile and $(b)$ the parabolic profile, where $\mathrm{Fr}=1.0$. 


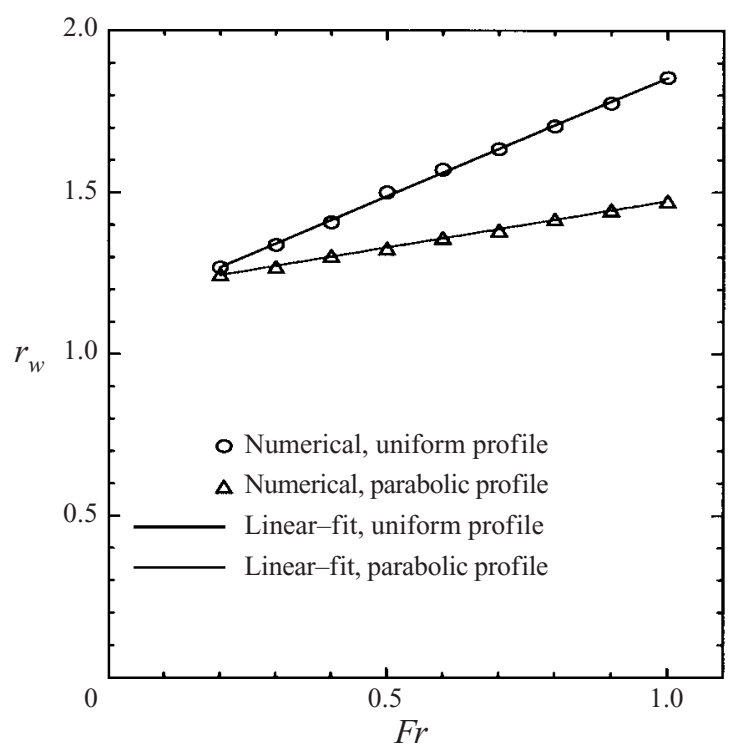

FIGURE 10. Fountain width $r_{w}$ plotted against $F r$.

where $\tau_{m}$ represents $\tau_{m i}, \tau_{m t}$ and $\tau_{m f}$ respectively and the constants $a_{0}$ and $a_{1}$ obtained by regression from the numerical results are listed in table 2 .

The reason for $a_{0} \neq 0$ is the same as that for $z_{m}$ as discussed in $\S 3.2 .2$.

\subsection{Fountain width}

When $\tau>\tau_{m f}$, the fountain flow is steady and a characteristic fountain width can be determined for each $F r$. Figure 9 presents the radial distribution of $v(r, z)$, the dimensionless vertical velocity, at different heights for both the uniform and parabolic profiles for $F r=1.0$. At each height, $v(r, z)$ decreases gradually as $r$ increases until a specific $r$ where $v(r, z)$ becomes zero for each $z$, which is the location where the upflow terminates and the downflow begins. Beyond this location $v(r, z)$ continues to reduce until a clearly defined minimum is reached, then increasing until it again crosses the zero line. The envelope of the minima for each height has itself a clearly defined minimum, corresponding to the $z=0.4 z_{m f}$ minimum, which occurs at $r \approx 1.9$ and $r \approx 1.45$ for $F r=1.0$ with the uniform and parabolic profiles respectively. The minimum of the envelope is clearly defined for all $\mathrm{Fr}$, and always corresponds to the $z=0.4 z_{m f}$ profile minimum. This width is defined to be the horizontal length scale $r_{w}$ which characterizes the fountain width at steady state and is nondimensionalized by $R_{0}$. This definition of $r_{w}$ corresponds very closely to the location of the fountain boundary temperature contours at the origin of the intrusion flow at $z=0.4 z_{m f}$. However, obtaining a consistent fountain width directly from the temperature contours is difficult owing to the smooth transformation from fountain to intrusion, whereas the very well-defined minimum in the envelope of the minima of the vertical velocity profiles makes the procedure straightforward.

Dimensional considerations show that $r_{w}$, like $z_{m}$, should be of the form $r_{w} \sim F r$. Figure 10 presents $r_{w}$ plotted against $F r$ for both the uniform and parabolic profiles with $0.2 \leqslant F r \leqslant 1.0$. It is clear that a linear relation exists between $r_{w}$ and $F r$, that is,

$$
r_{w}=a_{0}+a_{1} F r,
$$




\begin{tabular}{cccc}
\hline Profile & $a_{0}$ & $a_{1}$ & $R C$ \\
Uniform & $1.1228 \pm 0.0058$ & $0.7308 \pm 0.0088$ & 0.9995 \\
Parabolic & $1.1833 \pm 0.0024$ & $0.2864 \pm 0.0037$ & 0.9994 \\
TABLE 3. Regression results of (3.6) for both the uniform and parabolic profiles \\
\hline
\end{tabular}
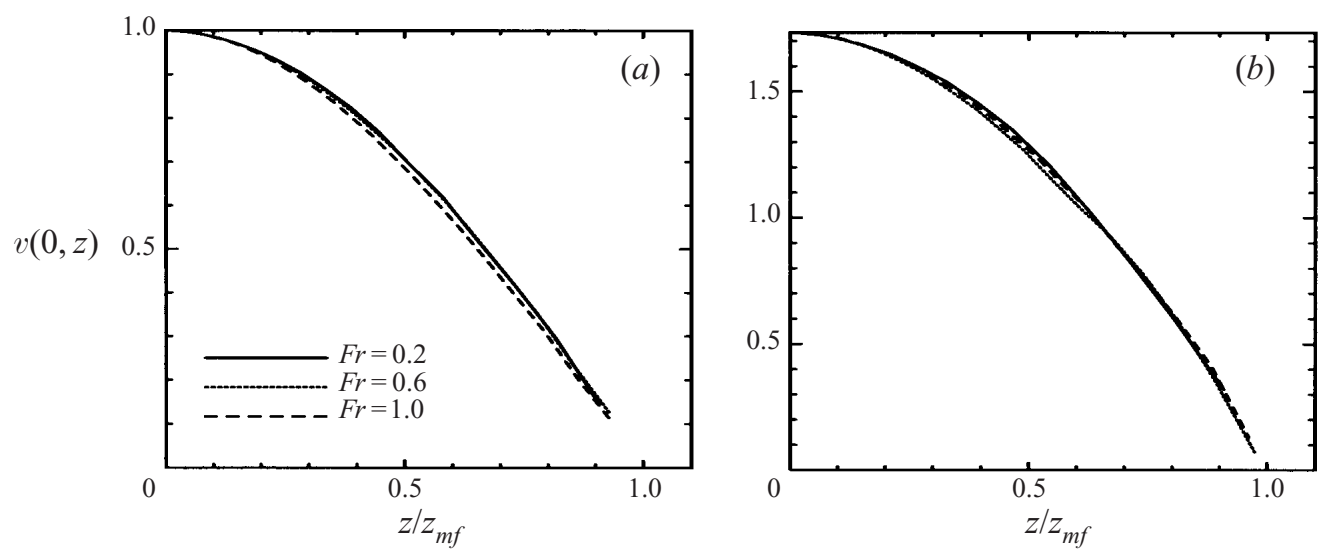

FIGURE 11. $v(0, z)$ plotted against $z / z_{m f}$ for a series of $F r$ with $(a)$ the uniform profile and (b) the parabolic profile at $\tau=10.0$.

with the values of $a_{0}$ and $a_{1}$ listed in table 3 , together with the regression coefficients.

The fountain core is the region bounded by $r=r_{w}$ and $z=z_{m f}$ and it is expected that these length scales can be used to parameterize the core flow. Further discussion of the core region is presented in $\S 4$.

\section{Steady flow patterns in the fountain core}

\subsection{Axial distribution}

\subsubsection{Axial velocity}

As discussed above, the vertical velocity of the fountain front on the symmetry axis, $v(0, z)$, reduces to zero when its position is at $z=z_{m f}$ at the steady state. Hence, $z_{m f}$ should provide the appropriate scaling for $v(0, z)$ in the $z$-direction, that is

$$
v(0, z)=f\left(\frac{z}{z_{m f}}\right) .
$$

The numerical results presented in figure 11 show the relation between $v(0, z)$ and $z / z_{m f}$ for $F r=0.2,0.6$ and 1.0 for both the uniform and parabolic profiles. Although only three sets of data are shown in the figure, all sets including those for other $\mathrm{Fr}$ in the range $0.2 \leqslant F r \leqslant 1.0$ are found to collapse onto a single curve. It is therefore clear that $z_{m f}$ does provide the appropriate length scale for the vertical velocity at the axis. Empirical tests showed that a cubic was the lowest-order polynomial to provide 

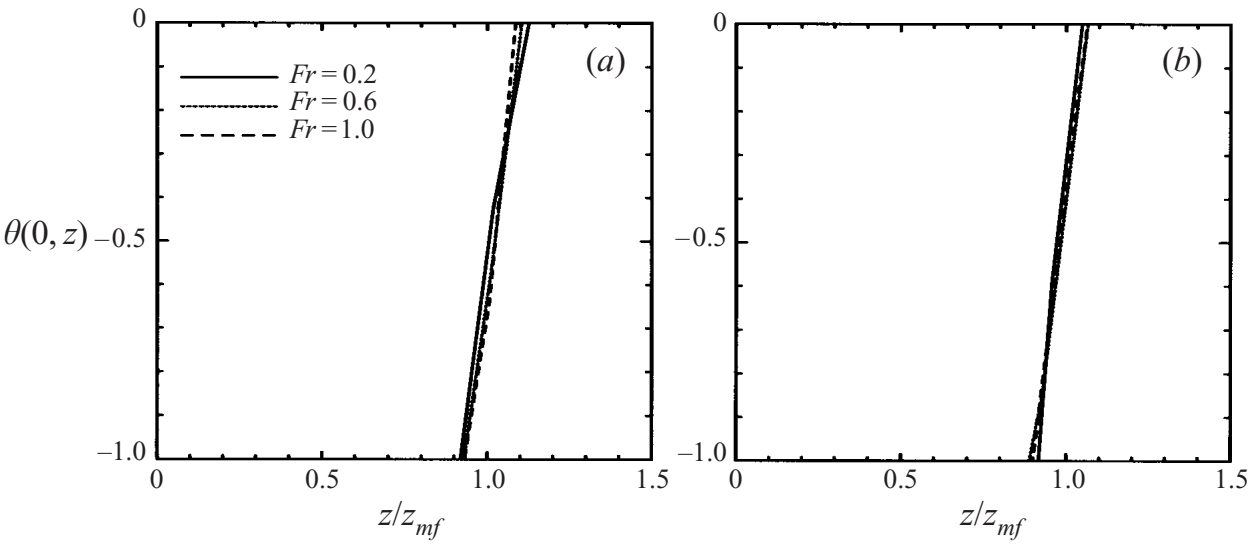

FIGURE 12. $\theta(0, z)$ plotted against $z / z_{m f}$ for a series of $F r$ with $(a)$ the uniform profile and $(b)$ the parabolic profile at $\tau=10.0$.
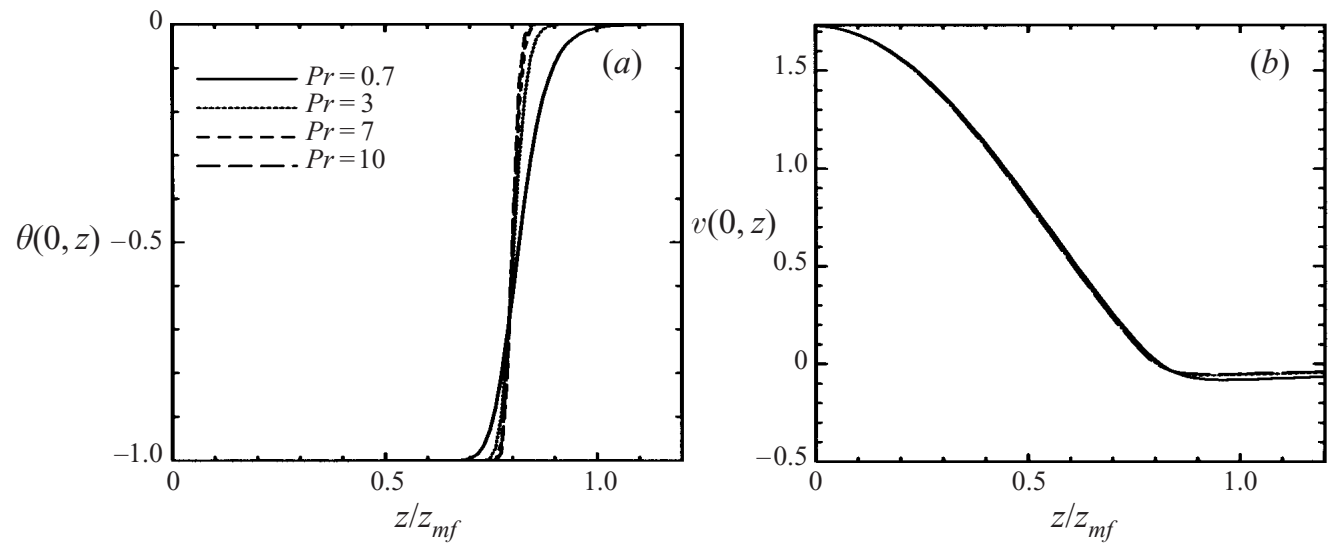

FIGURE 13. Axial distribution of $(a) \theta(0, z)$ and $(b) v(0, z)$ for different $\operatorname{Pr}$ for $F r=0.6$ with the parabolic profile.

a good fit to this relation, with the following explicit form for the uniform profile:

$$
v(0, z)=1.0000-0.0097\left(\frac{z}{z_{m f}}\right)-1.3341\left(\frac{z}{z_{m f}}\right)^{2}+0.3448\left(\frac{z}{z_{m f}}\right)^{3},
$$

and for the parabolic profile,

$$
v(0, z)=1.7321-0.0724\left(\frac{z}{z_{m f}}\right)-1.8402\left(\frac{z}{z_{m f}}\right)^{2}+0.1741\left(\frac{z}{z_{m f}}\right)^{3} .
$$

The squares of the regression coefficients of these two correlations are 0.9984 and 0.9977 respectively. There is no apparent physical basis for the use of a cubic for this relation; however it is clear that the cubic provides an excellent fit to the data.

\subsubsection{Axial temperature variation}

As shown in figure 4, numerical results reveal that the temperature variation occurs mainly in a very thin layer nearest the fountain front for both the unsteady and steady state when $\mathrm{Pr}=7$, indicating that there is little entrainment between the upflow and 

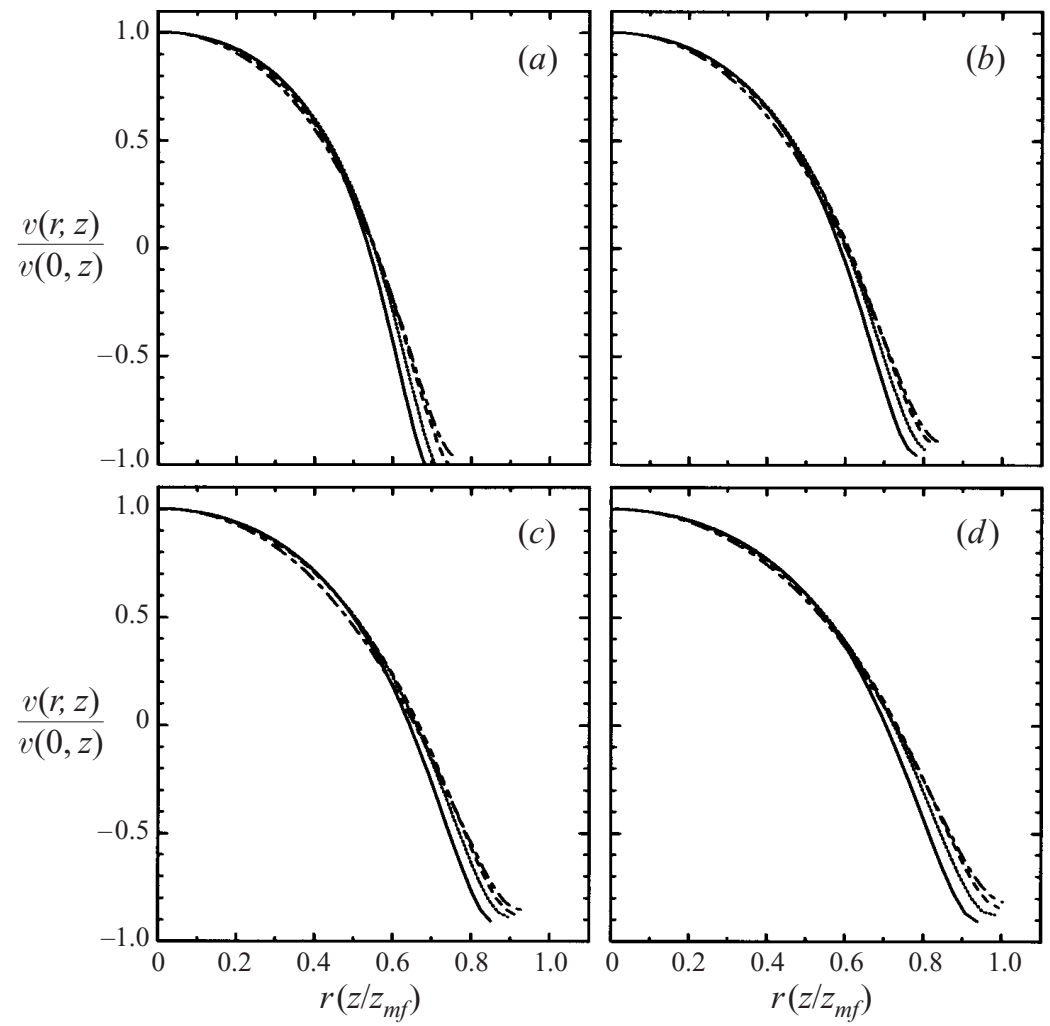

FIGURE 14. $v(r, z) / v(0, z)$ plotted against $r z / z_{m f}$ for $(a) F r=0.3 ;(b) F r=0.5 ;(c) F r=0.7$; and $(d)$ $F r=0.9$ with the uniform profile:,$- z / z_{m f}=0.6 ; \cdots \cdots, z / z_{m f}=0.65 ;----, z / z_{m f}=0.7$; - - - $z / z_{m f}=0.75$.

the surrounding ambient fluid. Figure 12 contains $\theta(0, z)$ plotted against $z / z_{m f}$ for $F r=0.2,0.6$ and 1.0 showing that, as expected, $z_{m f}$ is the appropriate length scale for $\theta(0, z)$, that is

$$
\theta(0, z)=f\left(\frac{z}{z_{m f}}\right) .
$$

\subsubsection{Influence of $\mathrm{Pr}$}

All the results presented so far have been for $P r=7$, which is the typical value for water at room temperature. When $\mathrm{Pr}$ becomes smaller, it is expected that the temperature variation should occur in a thicker layer nearest the fountain front. Figure 13 provides the evidence for this argument, presenting the temperature and velocity variations along the symmetry axis for $F r=0.6$ with the parabolic profile. It is also observed that the influence of $P r$ on the velocity profile is very small.

\subsection{Radial distribution}

\subsubsection{Zones for establishment and self-similarity}

The results in figure 9 show that above $z / z_{m f} \approx 0.55$ the fountain flow is fully established and for $0.55 \leqslant z / z_{m f} \leqslant 0.8$ a zone exists in which the vertical velocity is self-similar. For $z / z_{m f}>0.8$ the self-similarity gradually collapses until $z / z_{m f}$ reaches its maximum value of 1.0 . 

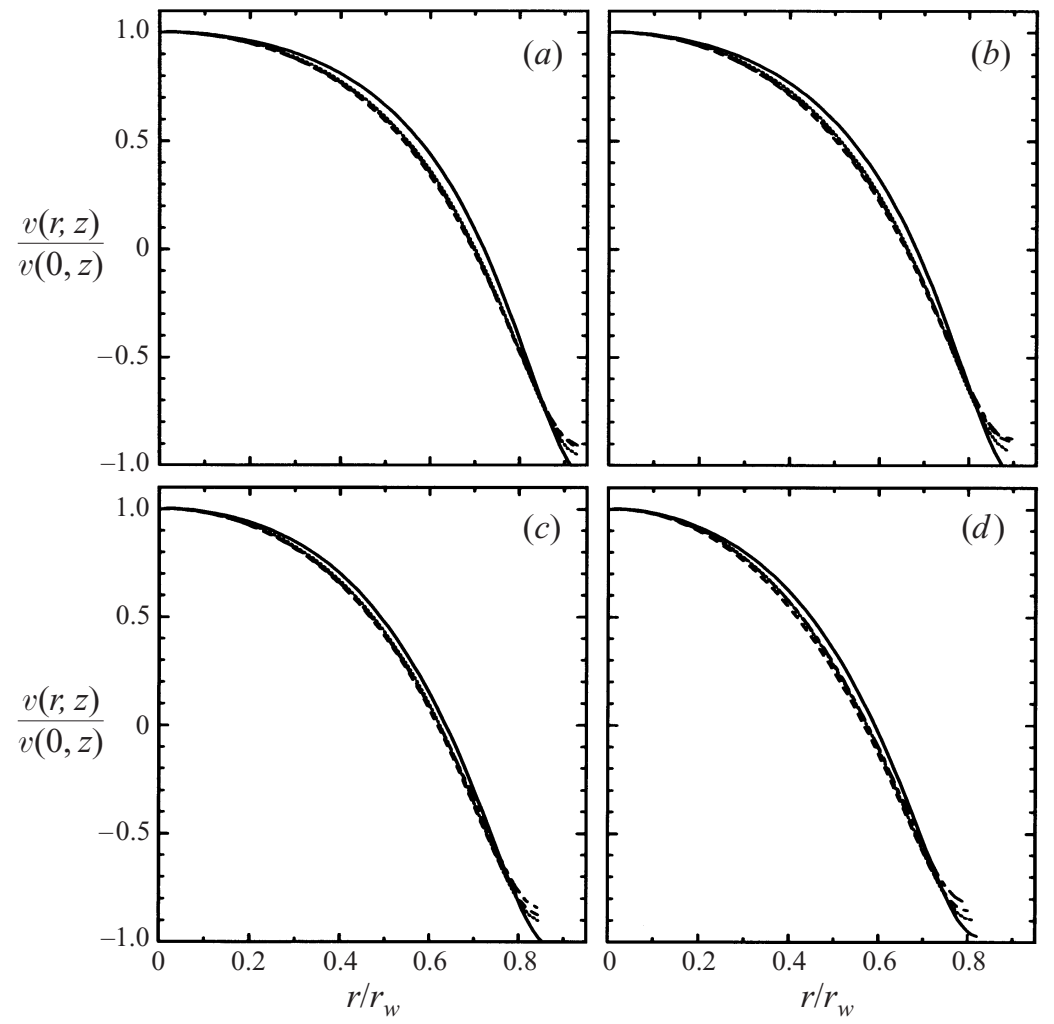

FIGURE 15. $v(r, z) / v(0, z)$ plotted against $r / r_{w}$ for $(a) z / z_{m f}=0.6 ;(b) z / z_{m f}=0.65 ;(c) z / z_{m f}=0.7$; and $(d) z / z_{m f}=0.75$ with the uniform profile:,$- F r=0.3 ; \cdots \cdots, F r=0.5 ;----, F r=0.7$; - -, $\mathrm{Fr}=0.9$.

\subsubsection{Radial distribution of vertical velocity in the zone of self-similarity}

In the zone of self-similarity, the height scale for the vertical velocity $v(r, z)$ should be $z_{m f}$, while the appropriate width scale is expected to be $r_{w}$. Hence, it is expected that a relation will exist between $v(r, z) / v(0, z), r / r_{w}$ and $z / z_{m f}$,

$$
\frac{v(r, z)}{v(0, z)}=f\left(\frac{r}{r_{w}}, \frac{z}{z_{m f}}\right) .
$$

To obtain an explicit form for (4.5), numerical simulations have been conducted for a series of $F r$. In figure $14 v(r, z) / v(0, z)$ is plotted against $r z / z_{m f}$ for several $z / z_{m f}$ and $\mathrm{Fr}$ with the uniform profile to determine the relation between $v(r, z) / v(0, z)$ and $z / z_{m f}$. Each set of results is collapsed onto a single curve by the $z / z_{m f}$ scaling. In figure $15, v(r, z) / v(0, z)$ is plotted against $r / r_{w}$ at fixed $z / z_{m f}$ for a range of $F r$, which shows that the $r_{w}$ scaling collapses the $F r$ variation in $v(r, z)$ onto a single curve at each $z$. Similar results are also obtained for the parabolic profile.

Figure 14 shows that $z / z_{m f}$ is the appropriate scaling for varying $z$ and fixed $\mathrm{Fr}$ while figure 15 shows that $r / r_{w}$ is the appropriate scaling for varying $\mathrm{Fr}$ and fixed $z$. To collapse all the $F r$ and $z$ results onto a single curve will require a combination of the $z_{m f}$ and $r_{w}$ length scales and therefore a general correlation should exist between $v(r, z) / v(0, z)$ and $r / r_{w} z / z_{m f}$. Figure 16 contains $v(r, z) / v(0, z)$ plotted against $r / r_{w} z / z_{m f}$ for a range of $\mathrm{Fr}$ and $z_{m f}$ values for both the uniform and parabolic profiles. The 

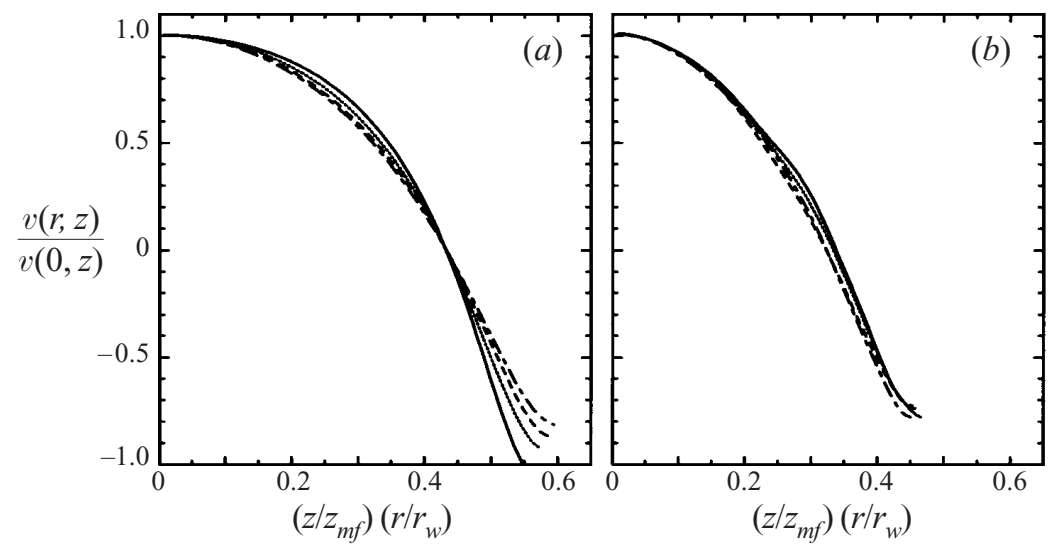

FIGURE 16. $v(r, z) / v(0, z)$ plotted against $\left(r / r_{w}\right)\left(z / z_{m f}\right)$ with $(a)$ the uniform profile and $(b)$ the parabolic profile: -,$F r=0.3$ and $z / z_{m f}=0.6 ; \cdots \cdots, F r=0.5$ and $z / z_{m f}=0.65 ;----$, $F r=0.7$ and $z / z_{m f}=0.7 ;-\cdot-, F r=0.9$ and $z / z_{m f}=0.75$.

combined $z_{m f}$ and $r_{w}$ scalings bring all the results very close to single curves. Again, a sixth-degree polynomial is the lowest-order polynomial which provides a good fit for each of the uniform and parabolic profiles, with the explicit form

$$
\begin{aligned}
\frac{v(r, z)}{v(0, z)}= & 1.017-1.269\left(\frac{r}{r_{w}} \frac{z}{z_{m f}}\right)+23.108\left(\frac{r}{r_{w}} \frac{z}{z_{m f}}\right)^{2} \\
& -220.711\left(\frac{r}{r_{w}} \frac{z}{z_{m f}}\right)^{3}+841.077\left(\frac{r}{r_{w}} \frac{z}{z_{m f}}\right)^{4} \\
& -1525.763\left(\frac{r}{r_{w}} \frac{z}{z_{m f}}\right)^{5}+1026.721\left(\frac{r}{r_{w}} \frac{z}{z_{m f}}\right)^{6}
\end{aligned}
$$

for the uniform profile and

$$
\begin{aligned}
\frac{v(r, z)}{v(0, z)}= & 1.010-0.502\left(\frac{r}{r_{w}} \frac{z}{z_{m f}}\right)+9.434\left(\frac{r}{r_{w}} \frac{z}{z_{m f}}\right)^{2} \\
& -231.568\left(\frac{r}{r_{w}} \frac{z}{z_{m f}}\right)^{3}+1239.703\left(\frac{r}{r_{w}} \frac{z}{z_{m f}}\right)^{4} \\
& -2962.975\left(\frac{r}{r_{w}} \frac{z}{z_{m f}}\right)^{5}+2588.980\left(\frac{r}{r_{w}} \frac{z}{z_{m f}}\right)^{6}
\end{aligned}
$$

for the parabolic profile. The square of the regression coefficients of these two correlations is 0.9949 and 0.9978 respectively. These two correlations, combined with (4.2) and (4.3), give a full description of the vertical velocity in the self-similar region of the weak fountain core at steady state.

\subsubsection{Radial distribution of horizontal velocity in the self-similar zone}

In the zone of self-similarity, the horizontal velocity $u(r, z)$ has a maximum value $u_{m}(z)$ for each height $z$. Similarly to $v(r, z)$ in the zone of self-similarity, the radial distribution of $u(r, z) / u_{m}(z)$ should be fully characterized by the two length scales $r_{w}$ and $z_{m f}$. Figure 17 shows $u(r, z) / u_{m}(z)$ plotted against $r / r_{w} z / z_{m f}$ for a range of $F r$ with 


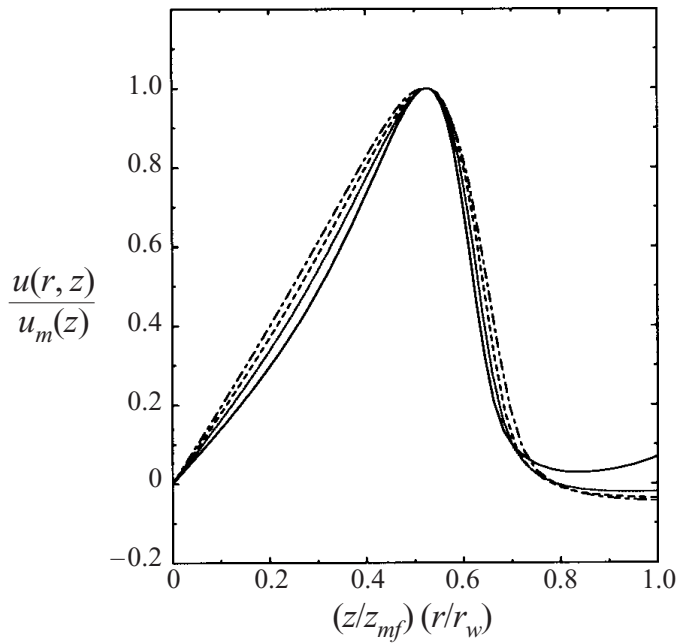

FIGURE 17. $u(r, z) / u_{m}(z)$ plotted against $\left(r / r_{w}\right)\left(z / z_{m f}\right)$ with the uniform profile profile: - , $F r=0.3$ and $z / z_{m f}=0.6 ; \cdots, F, F r=0.5$ and $z / z_{m f}=0.65 ;----, F r=0.7$ and $z / z_{m f}=0.7$; $-\cdot-, F r=0.9$ and $z / z_{m f}=0.75$.

the uniform profile. It is apparent that $r_{w}$ and $z_{m f}$ do provide a good parameterization, collapsing all the results close to a single curve for each of the inlet profiles.

\section{Conclusions}

We have presented a numerical study on the behaviour of weak axisymmetric fountains discharged upwards into a homogeneous environment. The numerical simulations have employed a time-accurate finite volume code which has been previously used for buoyancy-dominated and stratified flows. The behaviour of fountains with both uniform and parabolic discharge velocity profiles at the source has been investigated.

Qualitative information has been obtained from visualization of a typical time evolution of the transient temperature contours. Two distinct stages of evolution, that is the unsteady and steady stages respectively, have been identified at which the fountain flow has significantly different patterns.

The time series of the passage of the fountain front has been presented and the initial, temporary and final characteristic fountain heights have been determined quantitatively. With the dimensional and numerical results, explicit correlations between these maximum heights and $\mathrm{Fr}$, the Froude number defined at the source, have been determined. This confirms the results of the dimensional analysis and shows that the Froude number is the appropriate control parameter for this flow, and that the vertical length scale is a linear function of the Froude number. It has also been shown that the time scale for the development of the fountain flow is quadratic function of the Froude number and the Fr relation for the achievement of steady state has been obtained.

The final fountain height $z_{m f}$, which is non-dimensionalized by $R_{0}$, has been shown to be the appropriate length scale for the vertical velocity on the symmetry axis and, in combination with the fountain width $r_{w}$, which is also non-dimensionalized by $R_{0}$, to provide a complete parameterization of both the vertical and horizontal velocities in the fountain core in the region $0.55 \leqslant z \leqslant 0.8$, showing that in this region the flow 
is self-similar. Below the region of self-similarity is a zone of establishment, while above the region the self-similarity gradually collapses. The horizontal length scale, $r_{w}$, has been shown to be a linear function of the Froude number, again confirming the dimensional arguments.

Results presented in this paper were all obtained with $R e=200$; however additional results have been obtained with $200 \leqslant R e \leqslant 800$ to determine any $R e$ dependence. It has been found that there is very little $R e$ variation in the results, indicating that the weak fountain flow in this range of $R e$ is governed by a balance between buoyancy and advection as predicted by the dimensional analysis which indicates that $\mathrm{Fr}$ is the scale control parameter.

We are very grateful for the reviewers's comments which helped us to clarify some important points and improve the quality of the paper significantly. The financial support of an AusAID scholarship and from the National Natural Science Foundation and Yunnan Province of P. R. China to W. Lin is gratefully acknowledged.

\section{REFERENCES}

Abraham, G. 1967 Jets with negative buoyancy in homogeneous fluid. J. Hyd. Res. 5, 235-248.

ARMFIELD, S. W. 1991 Finite-difference solutions of the Navier-Stokes equations on staggered and non-staggered grids. Computers Fluids 20, 1-17.

ARMFIELD, S. W. 1994 Ellipticity, accuracy, and convergence of the discrete Navier-Stokes equations. J. Comput. Phys. 114, 176-184.

Armfield, S. W. \& Debler, W. 1993 Purging of density stabilized basins. Intl J. Heat Mass Transfer 36, 519-530.

Armfield, S. W. \& Patterson, J. C. 1992 Wave properties of natural-convection boundary layers. J. Fluid Mech. 239, 195-211.

Baines, W. D., Corriveau, A. F. \& Reedman, T. J. 1993 Turbulent fountains in a closed chamber. J. Fluid Mech. 255, 621-646.

Baines, W. D., Turner, J. S. \& Campbell, I. H. 1990 Turbulent fountains in an open chamber. J. Fluid Mech. 212, 557-592.

Bloomfield, L. J. \& KerR, R. C. 1998 Turbulent fountains in a stratified fluid. J. Fluid Mech. 358, 335-356.

Campbell, I. H. \& Turner, J. S. 1989 Fountains in magma chambers. J. Petrol. 30, 885-923.

Chen, C. J. \& Rodi, W. 1980 Vertical Turbulent Buoyant Jets. Pergamon.

Duffie, J. A. \& Beckman, W. A. 1991 Solar Engineering of Thermal Processes (2nd Edn). John Wiley \& Sons.

Fischer, H. B., List, E. J., Koh, R. C. Y., Imberger, J. \& Brooks, N. H. 1979 Mixing in Inland and Coastal Waters. Academic.

Huppert, H. E. \& Sparks, R. S. J. 1988 Melting the roof of a chamber containing a hot, turbulently convecting fluid. J. Fluid Mech. 188, 107-131.

LEONARD, B. P. 1979 A stable and accurate convective modelling procedure based on quadratic upstream interpolation. Comput. Meth. Appl. Mech. Engng 19, 59-98.

List, E. J. 1982 Turbulent jets and plumes. Ann. Rev. Fluid Mech. 14, 189-212.

Mizushina, T., Ogino, F., Takeuchi, H. \& Ikawa, H. 1982 An experimental study of vertical turbulent jet with negative buoyancy. Wärme- und Stoffübertragung 16, 15-21.

Morton, B. R. 1959 a Forced plumes. J. Fluid Mech. 5, 151-163.

Morton, B. R. $1959 b$ The ascent of turbulent forced plumes in a calm atmosphere. Intl J. Air Poll. 1, 184-197.

Patankar, S. V. 1980 Numerical Heat Transfer and Fluid Flow. Hemisphere.

Patterson, J. C. \& Armfield, S. W. 1990 Transient features of natural convection in a cavity. J. Fluid Mech. 219, 469-497.

RodI, W. 1982 Turbulent Buoyant Jets and Plumes. Pergamon. 
Seban, R. A., Behnia, M. M. \& Abreau, K. E. 1978 Temperatures in a heated air jet discharged downward. Intl J. Heat Mass Transfer 21, 1453-1458.

Turner, J. S. 1966 Jets and plumes with negative or reversing buoyancy. J. Fluid Mech. 26, 779-792. Turner, J. S. 1973 Buoyancy Effects in Fluids. Cambridge University Press.

TURNER, J. S. 1986 Turbulent entrainment: the development of the entrainment assumption, and its application to geophysical flows. J. Fluid Mech. 173, 431-471. 